\title{
El control interno de los inventarios: su incidencia en la gestión financiera de Due Amici Pizzería
}

\author{
María Consuelo Restrepo* \\ Candy Chamorro González ${ }^{* *}$ \\ Diego Carvajal Serna**
}

Recibido: 23 de junio de 2020

Aprobado: 24 de agosto de 2020

Restrepo, M., Chamorro, C., Carvajal, D. (2020). El control interno de los inventarios: su incidencia en la gestión financiera de Due Amici Pizzería. Revista Activos, 18(2), 137-163. https://doi.org/10.15332/25005278/6264

* Estudiante de X semestre de contaduría pública en la Universidad Católica Luis Amigó.

Correo electrónico: maría.restreposu@amigo.edu.co

** Contadora Pública por la Universidad de la Costa. Especialista en contabilidad internacional por la Universidad de Malta. Docente tiempo completo de la Universidad Católica Luis Amigó. Líder de la línea de investigación: contabilidad, ambiente y sociedad.

Correo electrónico: candiilorena@gmail.com.

ORCID: https://orcid.org/0000-0001-7332-8566

*** Contador Público. Especialista en mercadeo gerencial por la Universidad de Medellín. Docente de la Universidad Católica Luis Amigó.

Correo electrónico: diego.carvajalse@amigo.edu.co 


\section{Clasificación JEL: M41, M42}

\section{Resumen}

La gestión y el control del inventario son características cruciales para garantizar los segmentos financieros de las pequeñas y medianas empresas. Esta investigación está orientada a describir el manejo del control interno de los inventarios y su incidencia en la gestión financiera de la empresa Due Amici Pizzería. La metodología está contemplada desde el enfoque cualitativo y sustentada en un estudio de caso. Los resultados destacan que existen operaciones y mecanismos de entornos inciertos e imprecisos debido a la inexistencia formal de un sistema de inventarios en Due Amici Pizzeria. Así mismo, el restaurante presenta falencias en el establecimiento de políticas, lineamientos y procesos necesarios para un adecuado control interno de los inventarios; aquellas impiden mejores resultados en la gestión financiera de la empresa e inciden en la rentabilidad de la organización. Debido a esto, es pertinente generar estrategias ante los procesos más débiles de la organización.

Palabras clave: inventarios, control interno, gestión financiera. 


\title{
The internal control of inventories: its impact on the financial management of Due Amici Pizzeria
}

\begin{abstract}
Inventory management and control are crucial features to ensure the financial segments of small and medium enterprises. Therefore, the research is aimed at describing the management of internal control for inventory and its impact on the financial management of the company Due Amici Pizzeria. The methodology is based on a qualitative approach and supported by a case study. The results highlight that there are uncertain and imprecise operations and environment mechanisms due to the lack of a formal inventory system in Due Amici Pizzeria. Likewise, the restaurant has shortcomings in the establishment of policies, guidelines and processes necessary for an adequate internal control of inventories that hinder better results in the financial management of the company and affect the organization's profitability. Therefore, it is pertinent to generate strategies for the weakest processes of the organization.
\end{abstract}

Keywords: inventories; internal control; financial management. 


\section{Introducción}

El dinamismo del mercado actual es cada vez más competitivo, de ahí que las compañías constantemente se ven obligadas a implementar herramientas modernas de gestión empresarial y contables con el propósito de hacer posible el cumplimiento de sus objetivos y metas, pensando siempre en obtener mayores garantías de permanencia en el mercado a largo plazo (Aristizabal y Guzmán, 2017).

En ese contexto, los inventarios son reconocidos como un elemento clave dentro de los factores de competitividad que toda organización debe gestionar, incluyendo las pymes (Serna y Rivera, 2018). Sin embargo, la gestión y el control de los inventarios son algunas de las funciones más complejas a nivel empresarial, dado que implican establecer políticas, lineamientos y el diseño de herramientas de control para mantener existencias, con el objetivo de garantizar la fidelidad de los clientes y la protección ante incertidumbres que puedan ser afrontadas a menor costo (Peña, y Oliveira, 2016).

En este punto, se reconoce el eje problematizador de las pymes debido a que presentan grandes dificultades con el manejo de sus inventarios (Castellanos, 2017), desconociendo que en ese proceso se encuentran las fuentes de ingresos más importantes de la compañía, en términos de mercaderías. Además, fortalece la gestión empresarial de dichas organizaciones, "que cobra relevancia en su crecimiento, desarrollo y sostenibilidad" (Pereira, 2019, p. 40).

Así, el presente trabajo destaca en el entorno investigativo porque su intención radica en describir la filosofía contable en el sistema del control interno de los inventarios y la gestión financiera del restaurante Due Amici Pizzería. Permitiendo, de esa manera, caracterizar los elementos que resaltan el buen manejo de los inventarios y la generación de estrategias ante los procesos más débiles de la organización. Finalmente, el estudio indicará si los procesos que implementa el restaurante en materia de inventarios han impactado en la gestión financiera o en la rentabilidad. 
El control interno de los inventarios: su incidencia | REVISTA ACTIVOS 141 en la gestión financiera de Due Amici Pizzería

\section{Referente teórico}

\section{Control interno de los inventarios}

Los inventarios juegan un papel importante en las empresas del sector comercial, industrial y de servicio, dado que la permanencia de estas organizaciones en el mercado, nacional o internacional, está ligada al buen manejo y control de ellos. Es así como, de acuerdo con Godoy (2014), los inventarios se definen bajo las Normas Internacionales de Contabilidad (NIC) como activos que se adquieren con el objeto de ser vendidos en el transcurso de la operación; pero, también se entienden como aquellos que están en proceso de producción y que tienen como fin ser vendidos, dispuestos para el consumo de producción o para la prestación de un servicio.

Sumado a eso, Godoy (2014) expresa que los inventarios que no se aprecian desde las NIC se definen como: "todos aquellos derechos que posee el ente económico, representados en materia prima, productos en proceso y producto terminado, transformado o no por el ente económico, que van a ser utilizados en el desarrollo del objeto social” (p. 330).

Paralelamente, se considera importante documentar una breve línea de tiempo que da cuenta del origen del inventario; este permite identificar que, desde los inicios del hombre, el individuo ha sobrevivido con todos los recursos proporcionados por la naturaleza, los cuales fueron escaseando con el transcurrir de los años. A partir de esos datos, se reconoce que dentro de la época del nomadismo los hombres se vieron en la necesidad de salir en la búsqueda de alimentos, crear refugios y diseñar estrategias para adquirir y conservar los recursos que les servía como medios de subsistencia. De esta manera, nacen las distintas épocas o momentos del mercantilismo y de ahí el perfeccionamiento del manejo y control de los inventarios (figura 1). 
Figura 1. Línea de tiempo de inventarios

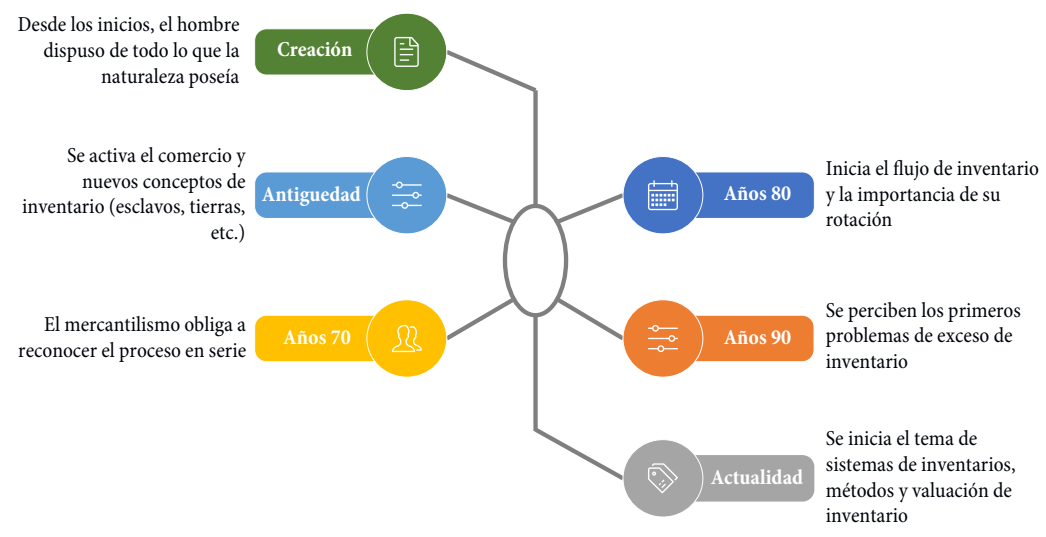

Fuente: elaboración propia.

Dadas las diferentes acepciones que se le da a los inventarios, se reconoce la importancia de estos activos para las diferentes empresas. Sin embargo, el proceso de los inventarios debe ser un lineamiento intrínseco que permita el desarrollo del objeto social de toda empresa; por tanto, es necesario contar con unas herramientas que coadyuven a implementar un control riguroso sobre estos. De esta manera, las empresas dedicadas al servicio en la satisfacción de necesidades de consumo deben gestionar la implementación de sistemas de inventarios que sean capaces de proporcionar indicaciones sobre las variaciones sutiles y a gran escala, que probablemente ocurran en la cadena de suministro y que impactan naturalmente en la toma de decisiones (Díaz y Tubón, 2018; Kumar et al, 2017).

Es por ello que, el control de los inventarios debe regularse a través de políticas, lineamientos y herramientas contables que permitan reconocerlos como activos poseídos para la venta en el curso normal de la operación; en proceso de producción de cara a esa venta o en forma de materiales o suministro. De esta manera, se logra que puedan ser consumidos en el proceso de producción o en la prestación de servicios (Carvalho, 2009; Ariza y Villasmil, 2014). 
Ante ello, es importante revelar la definición del control interno de acuerdo con la Ley 87 de 1993, artículo 1:

Sistema integrado por el esquema de organización y el conjunto de los planes, métodos, principios, normas, procedimientos y mecanismos de verificación y evaluación adoptados por una entidad, con el fin de procurar que todas las actividades, operaciones y actuaciones, así como la administración de la información y los recursos, se realicen de acuerdo con las normas constitucionales y legales vigentes dentro de las políticas trazadas por la dirección y en atención a las metas u objetivos previstos (Ley 87, 1993).

Lo anterior permite reconocer la importancia de este proceso en la organización puesto que facilita la continuidad del proceso productivo y la satisfacción de la demanda de los clientes (Úbeda, 2013). Así mismo, el control interno de los inventarios actúa como regulador o amortiguador entre los ritmos de salida de una fase y los ritmos de entrada de las siguientes (Ávila, 2010). Además de esto, Cepeda (2002) expresa que el control interno significa comprobación, intervención o incluso inspección de las diferentes organizaciones con el fin de apoyar su desarrollo y capacidad de permanecer en el mercado, es decir, sirven en la creación de bases que soportan o incentivan la búsqueda de resultados esperados.

Mora (2016) desarrolló una de las propuestas de control de los inventarios: a través de formulaciones metodológicas, el autor generó los lineamientos adecuados para optimizar los inventarios por medio de una estructura sistemática de fundamentación teleológica, la cual consiste en optimizar los tiempos tanto de entrega y salida de la mercancía, al almacén. De esta manera, Mora (2016) propone un sistema de inventarios representado en cuatro niveles: (1) nivel de orden funcional, alusivo al proceso instrumental del almacenamiento; (2) nivel de orden operacional, también llamado orden de las dos acciones y operaciones factibles con los inventarios; (3) nivel de orden táctico que se alimenta de la pregunta “¿cómo se deben hacer las cosas?”; (4) nivel de orden estratégico, donde se generan las estrategias para 
alcanzar el sistema estructural propuesto, el cual consiste en la satisfacción de cantidad y oportunidad del inventario en sus distintas áreas.

Desde otro enfoque, Ramírez y Ramos (2016) enfatizan el sistema de control de inventarios justo a tiempo, considerado como otra forma de controlar los inventarios de las empresas, ligado a su objeto social. El sistema justo a tiempo es un método cíclico que consiste en la fijación de metas que apuntan a productos de calidad y puntualidad. De esa manera, se alcanzan los beneficios esperados en los que se perciba un aumento de competitividad, capacidad con los mínimos recursos, fortalecimiento de la industria, etc.

Según Nahmias (2014) los modelos de control de inventarios sujetos a incertidumbre son básicamente de dos tipos: la revisión periódica (el nivel del inventario se conoce solo en puntos discretos del tiempo) y la revisión continua (se tiene conocimiento todo el tiempo). Adicionalmente, Cuervo, Osorio y Duque (2016) explican que uno de los sistemas de control de inventarios más eficaces es el modelo $\mathrm{ABC}$ que busca mejorar la fluidez de los productos por medio de la sistematización de controles y clasificación de los bienes o servicios que mejoran la gestión de existencia en inventarios.

A partir de los argumentos precedentes, la tabla 1 compila los distintos procesos de control de inventarios que las empresas pueden implementar para mejorar y optimizar sus actividades de control, debido a que se reconoce que estos sistemas de inventarios son la pieza clave para el buen desempeño de la organización y la credibilidad en el mercado.

Se concluye que el control interno juega un papel de vital importancia dentro de las organizaciones, pues estas deben establecer unas políticas acordes a su objeto social en las cuales se determinen responsabilidades para cada uno de los departamentos con el objetivo de alcanzar las metas de la organización y el bienestar de todos sus colaboradores. 
El control interno de los inventarios: su incidencia en la gestión financiera de Due Amici Pizzería

Tabla 1. Procesos que contemplan el control interno de los inventarios

\begin{tabular}{|c|c|c|}
\hline \multicolumn{2}{|c|}{ Control Interno de los Inventarios } \\
\hline $\begin{array}{c}\text { Optimización de } \\
\text { inventarios }\end{array}$ & Justo a tiempo & Modelo ABC \\
\hline $\begin{array}{c}\text { Sistematizar la entrada y } \\
\text { salida de productos del } \\
\text { almacén }\end{array}$ & $\begin{array}{c}\text { Ejercer un sistema cíclico } \\
\text { y evitar un mínimo de } \\
\text { desperdicios }\end{array}$ & $\begin{array}{c}\text { Organizar los productos } \\
\text { por su importancia }\end{array}$ \\
\hline $\begin{array}{c}\text { Aplicar niveles de orden } \\
\text { funcional y operacional }\end{array}$ & $\begin{array}{c}\text { Fijar metas que apunten a } \\
\text { la producción de calidad }\end{array}$ & $\begin{array}{c}\text { Satisfacer necesidades } \\
\text { y minimizar los costos }\end{array}$ \\
\hline \multicolumn{3}{|c|}{ Pasos para su implementación } \\
\hline $\begin{array}{c}\text { (1) Actualización constante } \\
\text { de la información }\end{array}$ & $\begin{array}{c}\text { (2) Monitorear } \\
\text { en tiempo real }\end{array}$ & $\begin{array}{c}\text { (3) Establecer días } \\
\text { de inventarios }\end{array}$ \\
\hline \multicolumn{2}{|c|}{ (4) Calcular máximos y mínimos de puntos de orden } \\
\hline
\end{tabular}

Fuente: elaboración propia a partir de Mora (2016), Nahmas (2014) y Perdomo (2000).

\section{Incidencia del control interno de los inventarios en la gestión financiera}

La gestión financiera es una actividad encargada de planificar, organizar, dirigir, controlar, monitorear y coordinar todo el manejo de los recursos financieros con el fin de generar mayores beneficios o resultados (Mora y Espinosa, 2018). En este sentido, el propósito es lograr que "la organización se desenvuelva con efectividad, apoyar a la mejor toma de decisiones financieras y generar oportunidades de inversión para la compañía" (Terrazas, 2009, p. 57).

No obstante, Rojas, Guisao y Cano (2011) definen la gestión de inventarios como el acto de organizar, planificar y controlar el conjunto de existencias pertenecientes a una organización. Pero, aseguran que organizar significa fijar criterios y políticas para su regulación con el objetivo de determinar las cantidades más convenientes de cada uno de los artículos; 
dicha gestión permite, adicionalmente, establecer métodos de previsión, orientados a identificar los tiempos y cantidades de reposición con el fin de controlar los movimientos de entradas y salidas, el valor del inventario y las tareas a realizar.

Por lo tanto, el control interno de los inventarios tiene como finalidad hacer un análisis de fondo a las políticas de gestión de la empresa sobre su manejo: la existencia de los proveedores, su ingreso al almacén, el registro en la planilla o sistema, exhibición, las ventas, entre otros elementos (Zhong et al, 2020). Lo anterior permitirá ejecutar los cambios pertinentes y evitar la pérdida del inventario en sus distintas modalidades; además, remarca la importancia de hacer un seguimiento de los empleados en sus diferentes actividades dentro de la empresa, donde el vínculo entre los inventarios y los clientes es relevante para su buen desempeño comercial.

Así, el control de los inventarios debe enfocar estrategias de cumplimientos a través de las políticas establecidas por el departamento de gestión financiera de la organización. Este departamento se encarga de direccionar los procesos, procedimientos, técnicas y prácticas encaminadas al manejo correcto y efectivo de los recursos financieros de la entidad (Gitman, 2016; Biswas et al, 2017).

En ese contexto, el control interno de los inventarios deberá apoyarse en unas políticas sólidas acordes a los intereses financieros de la empresa u organización; puesto que desde allí se dan las pautas para que los sistemas de control interno de la organización direccionen sus prácticas organizacionales en un tiempo razonable y sin afectar su objeto social.

Entre tanto, Wang et al. (2019) reconocen que los inventarios representan una variable de decisión importante en todas las etapas de fabricación, distribución y venta de productos. Indudablemente, estas decisiones afectan la gestión financiera de la organización, ya que un inadecuado control interno de estos activos podría reducir el nivel de rentabilidad de la organización. Por lo tanto, una entidad siempre deberá garantizar que el inventario esté listo, organizado y sistematizado. 
En este sentido, es importante que las empresas implementen y mantengan buenos sistemas de control de inventario, como la cantidad de orden económico y el justo a tiempo. Consiguiendo, de esta forma, que las empresas mantengan niveles ideales de inventarios y que, a su vez, permitan aumentar la rentabilidad de las organizaciones.

\section{Materiales y métodos}

Con el objetivo de profundizar en la problemática que afronta la empresa a partir de su entorno o realidad interior, la investigación se apoya en un enfoque cualitativo sustentado en un estudio de caso (Chenhall y Smith, 2011). Entonces, este trabajo posee un alcance descriptivo abordado desde el método deductivo, que emplea principios e informaciones verídicas para detectar particularidades asociadas al estudio en curso (Hernández et al., 2014).La población de estudio estuvo conformada por el restaurante Due Amici Pizzería, ubicado en la ciudad de Medellín (Antioquia). La empresa lleva seis años de funcionamiento y su objeto comercial está enfocado en la elaboración de pizzas, sándwiches, galletas y postres.

Por un lado, el procedimiento, desde el punto de vista de las herramientas, las técnicas y las metodológicas utilizadas, estuvo dado inicialmente a través de fuentes primarias, sustentadas en diferentes artículos, libros, trabajos de grado, entre otros documentos relacionados con el control interno de los inventarios y gestión financiera.

Por otro, se implementaron diversas fuentes secundarias, entre ellas: (1) la documentación proporcionada por la empresa como la facturación, los movimientos registrados en el software contable, el reporte de la entrega de proveedores y otros documentos adicionales; (2) se realizó una entrevista de catorce preguntas dirigidas al administrador con el objetivo de tener mayor precisión y compresión de las actividades relacionadas con el inventario; (3) se aplicó la unidad de observación en donde estuvieron involucrados el cocinero, meseros y otro personal relacionado con el inventario. 
El análisis de la información se llevó a cabo a través de la herramienta Excel que permitió organizar y sistematizar las respuestas o hechos observados, estos, a su vez, permitieron una contrastación de la base teórica contable a partir de los resultados hallados en el estudio.

\section{Resultados}

El objetivo principal de esta sección es ilustrar los hallazgos relacionados con la temática de estudio en el ámbito de la disciplina contable. Los resultados permiten analizar la importancia del control de los inventarios y cómo esta puede contribuir a perpetuar los problemas financieros o incrementar la liquidez o competitividad de la empresa.

\section{Control interno de los inventarios en Due Amici Pizzería}

Los elementos del sistema de control interno de los inventarios se dividen en tres partes: objetivos, políticas y procesos (Weber et al., 2018). Sin embargo, Shenoy y Rosas (2018) manifiestan que las pequeñas empresas deberían, por lo menos, tener objetivos bien trazados asociados al mínimo de existencias en materia prima y de producto terminado, con el fin de tener una capacidad ante la situación de consumo de los clientes.

En ese orden de ideas, la primera pregunta del análisis estuvo enfocada en saber si la empresa contaba con un sistema de control interno de los inventarios. En este caso, el restaurante no tiene un sistema de apoyo que permita a los empleados un pleno conocimiento, de forma ordenada $y$ sistemática, para el buen manejo y control de los inventarios, por lo que aquellos se enfrentan a esta carencia a diario. Debido a ello, la manipulación y el cuidado de los productos y los materiales se lleva a cabo por medio de una distribución de tareas basadas en el conocimiento empírico que el administrador adquirió desde los inicios de la empresa. 
No obstante, el cocinero principal implementó la herramienta Google Drive con el fin de registrar el inventario y sus proveedores. De tal manera, como la figura 2 revela, el $80 \%$ de las políticas del manejo del control interno de los inventarios se da de forma oral (basados en el empirismo), mientras el $20 \%$ de ellas es de manera escrita, sin el rigor técnico contable. Sumado a esto, es preciso remarcar que el $65 \%$ de las responsabilidades del manejo del control interno de los inventarios recae sobre el administrador y el otro $35 \%$ en el cocinero (figura 3 ).

Figura 2. Manejo del inventario

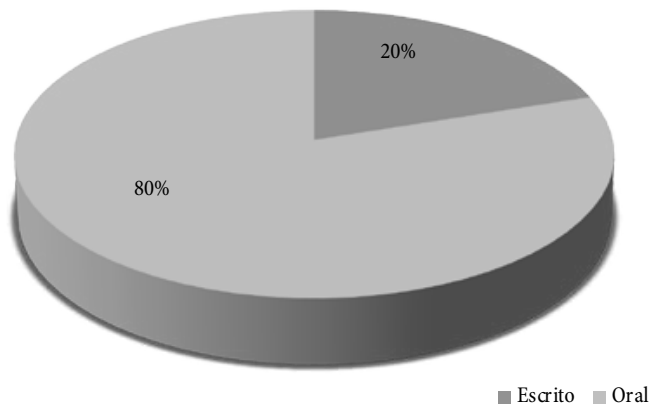

Fuente: elaboración propia.

Figura 3. Custodia inventarios

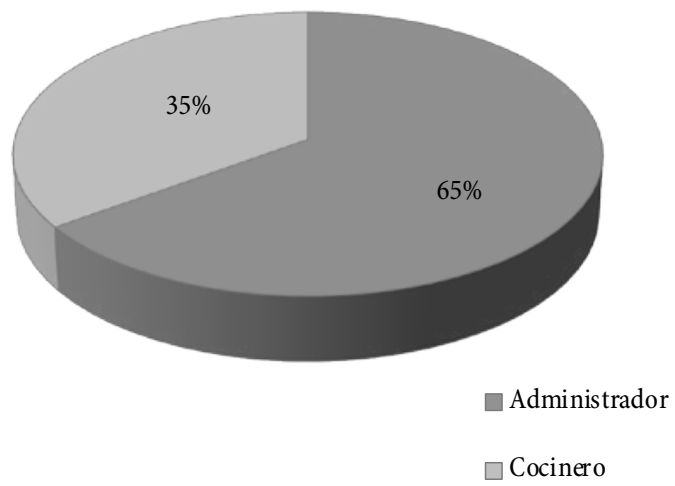

Fuente: elaboración propia. 
Con el fin de conocer el proceso que garantiza un nivel de existencias adecuado en los inventarios, fue importante saber los lineamientos o políticas pertinentes para los proveedores, por lo cual surgieron las preguntas: ¿se hace un estudio previo de proveedores?, ¿cómo se lleva a cabo su selección?, ¿cuántos proveedores tienen? En este sentido, la organización manifestó que no tiene políticas de selección de proveedores, aunque, para ellos prima que los productos suministrados sean de calidad y sirvan adecuadamente para sus preparaciones saludables.

La figura 4 detalla que la empresa cuenta con diecinueve proveedores y diez de ellos vienen prestando el servicio desde hace seis años (marcados en color gris). Además, cinco de ellos (Bufalagrande, Colbufala, Setas, Sierra Maestra y la Minorista) son esenciales para el desarrollo de la actividad económica del restaurante.

Figura 4. Permanencia de proveedores

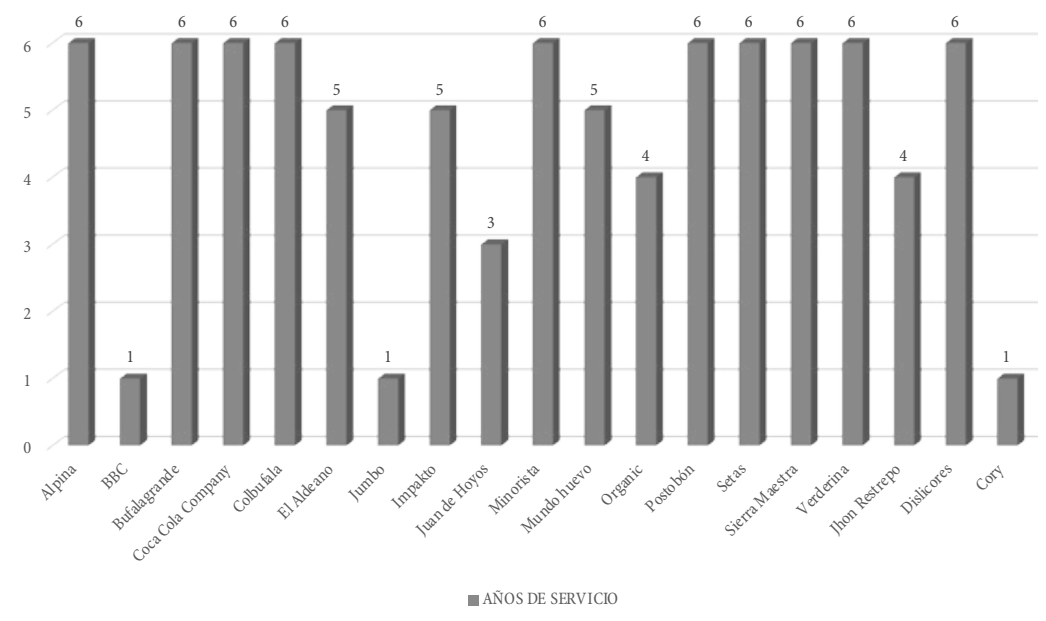

Fuente: elaboración propia.

$\mathrm{Al}$ respecto, es conveniente identificar la frecuencia de compras de materia prima (insumos para la transformación), para ello se formularon las preguntas: ¿tienen estipulado cada cuánto la empresa debe hacer 
pedidos?, ¿ cómo es el proceso?, ¿quién es la persona encargada de realizar las órdenes de compra? Se determinó que las mayores compras se realizan de forma semanal, quincenal y mensual en un $33.33 \%$, $20.37 \%$ y $22.22 \%$ respectivamente. El resto de estas son bimestrales en un $9.26 \%$, trimestrales en un $1.85 \%$ y semestrales en un $3.70 \%$, estos últimos porcentajes demuestran que dichos insumos son fáciles de almacenar o de manipular para alcanzar el producto final que la empresa pretende conseguir para ofrecer a los clientes (figura 5). Además de eso, en este caso, se comprobó que el cocinero es la persona responsable de los pedidos.

Figura 5. Frecuencia de compras

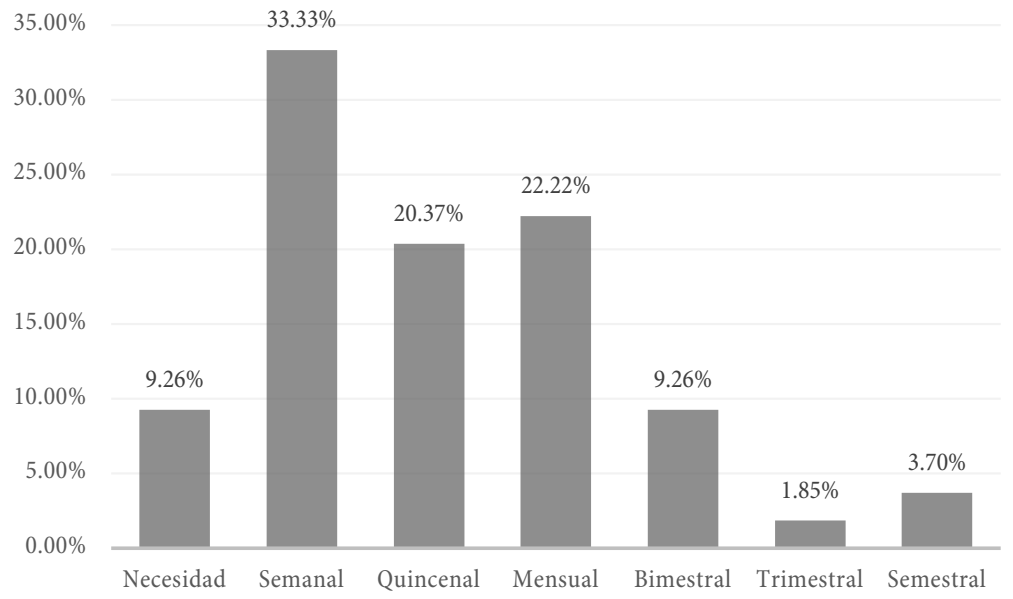

Fuente: elaboración propia.

Cabe resaltar que la frecuencia de compras de materia prima (MP), está asociada a la capacidad de almacenaje que la empresa Due Amici Pizzería tiene en su local. Así, se evidencia que el restaurante tiene la facultad de almacenar el $94 \%$ de los insumos que compra; sin embargo, no puede almacenar el $6 \%$ restante puesto que el espacio del local es reducido (figura 6). 
Figura 6. Almacenamiento de MP

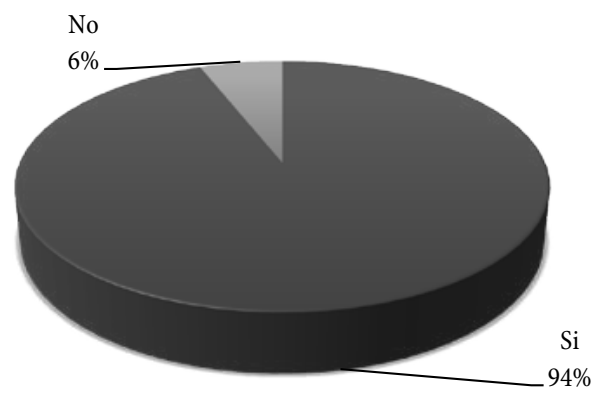

Fuente: elaboración propia.

Del mismo modo, es necesario hacer alusión del almacenaje que se lleva a cabo con el producto terminado (Рт) y listo para la venta. La figura 7 muestra cómo el $50 \%$ de dichos productos no se almacenan debido a que se deben ser producidos para el consumo inmediato. Sin embargo, el otro $50 \%$ sí requiere almacenamiento, como son los acompañamientos extras (tinto, mocaccino, refrescos, etc.) cuyo fin es amenizar el ambiente.

Figura 7. Almacenamiento de PT
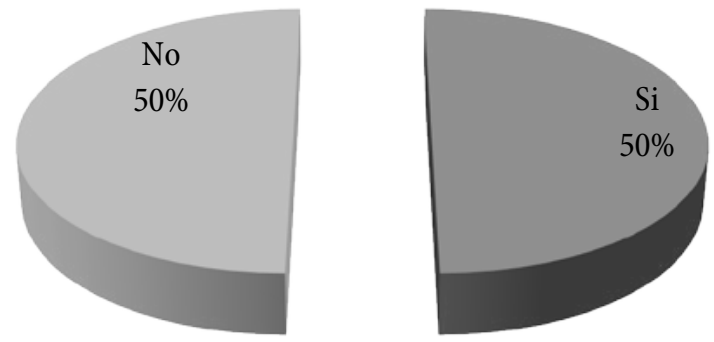

Fuente: elaboración propia.

Además de lo anterior, la figura 8 revela cómo es el sistema de protección del inventario en el restaurante Due Amici Pizzería. Dos personas están encargadas de dicha tarea: primero, el cocinero tiene como misión hacer el 
conteo físico del inventario. Este se organiza en cuatro momentos: supervisar la materia prima de acuerdo con el método Peps; hacer la respectiva separación; listar en un tablero los productos que se requieren comprar; $y$, finalmente, contar las existencias de las diferentes zonas, dejando la evidencia en su sistema de apoyo (Google Drive). En segundo lugar, el administrador verifica que se haya llevado a cabo la actividad numérica a cabalidad, y, a partir de su cumplimiento, prosigue con la toma de decisiones, de acuerdo con la experticia que ha adquirido en el negocio.

Figura 8. Resguardo del inventario

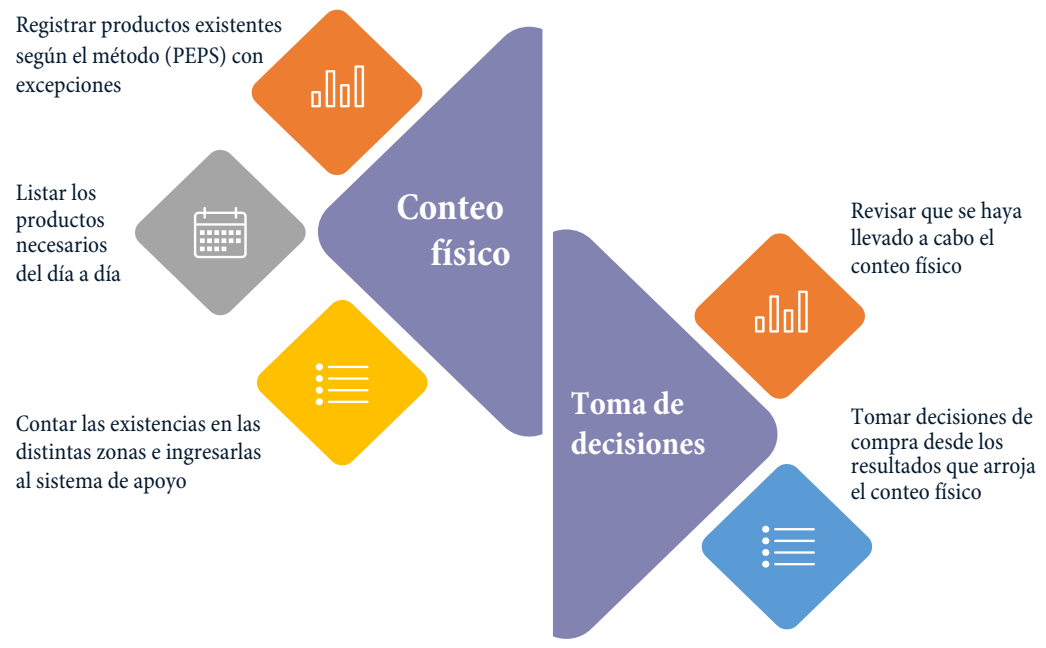

Fuente: elaboración propia.

Por consiguiente, es importante abordar la pregunta: ¿cuáles son los pasos que realizan para la compra de insumos? En este contexto, es importante señalar que el administrador es quien lleva a cabo la solicitud de compra y le da el visto bueno; igualmente, realiza como máximo tres cotizaciones a sus proveedores, en el caso de ser necesario, debido a que para ciertos insumos solo cuenta con un proveedor (figura 9). 
Figura 9. Proceso de compra y recepción de la compañía

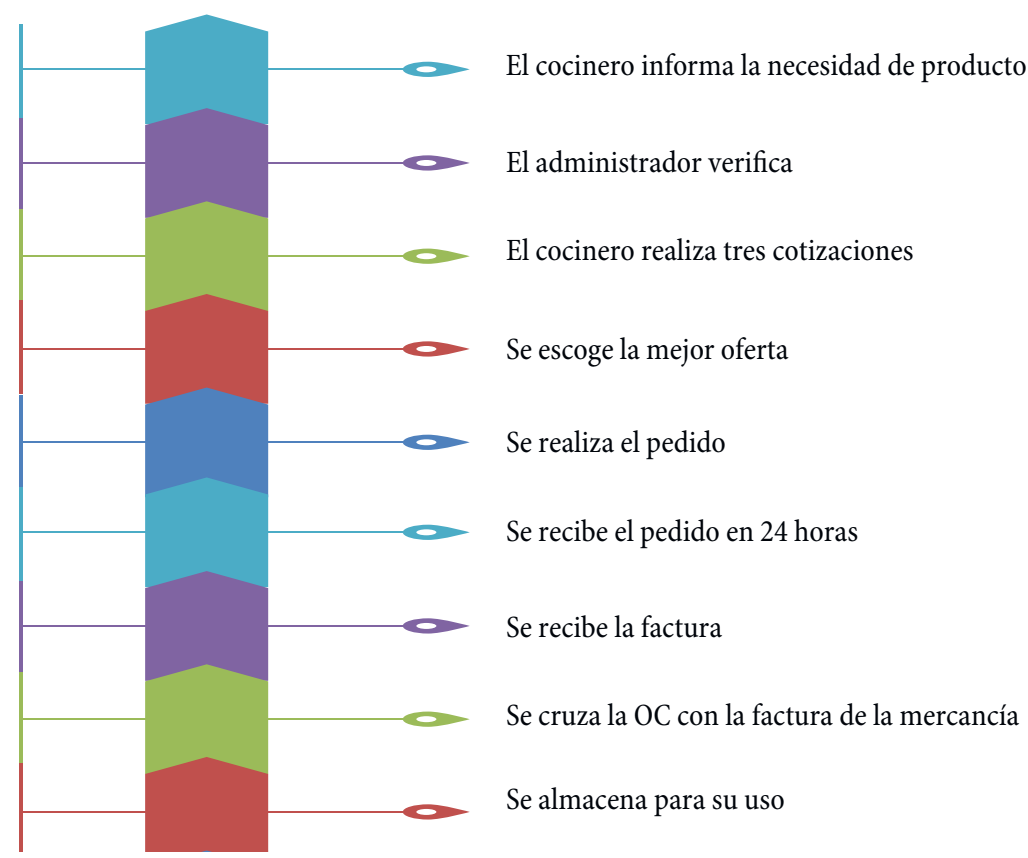

Fuente: elaboración propia.

\section{Incidencia en la gestión financiera de Due Amici Pizzería}

Si se entiende la gestión financiera como el acto de fijar políticas y controles para la coordinación de los recursos financieros de la organización, es posible afirmar que la empresa Due Amici Pizzería no planifica, ni organiza, ni monitorea actividades encaminadas a la gestión financiera de la empresa.

Ahora bien, teniendo en cuenta que la finalidad de esta gestión es generar niveles superiores de rentabilidad en cada periodo contable, fue importante identificar el comportamiento de los movimientos financieros del último semestre del año 2019. Lo anterior permitió determinar las 
características que inciden o no en los recursos financieros del restaurante Due Amici Pizzería debido a la inexistencia de un sistema de control interno de los inventarios.

En ese sentido, fue importante realizar preguntas relacionadas con las compras, gastos y ventas de la organización. De acuerdo a ello, la figura 10 demuestra que la empresa obtuvo unas ventas de 247250500 cop, para unas compras de 14500000 cop y unos gastos totales 53366000 cop, eflejando una rentabilidad de $179384500 \mathrm{cop}$ en el segundo semestre de 2019.

Figura 10. Utilidad del segundo semestre del 2019

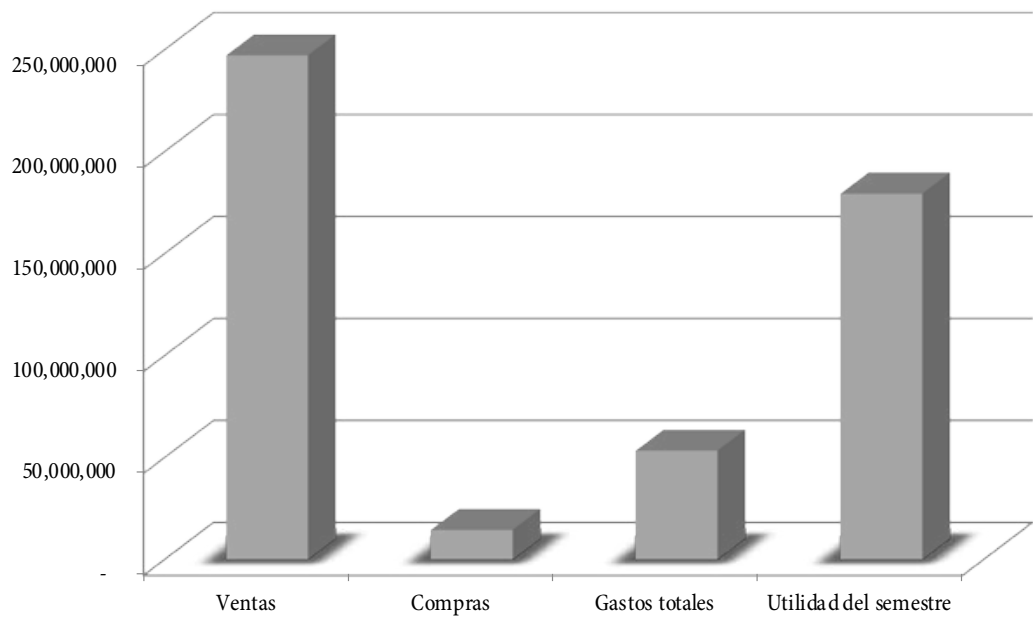

Fuente: elaboración propia.

La figura 11 arroja resultados acerca de las ventas y las utilidades presentadas en el segundo semestre del 2019, con su respectivo porcentaje. Siendo así, se percibe que para los meses de julio, octubre y diciembre se obtienen ventas mayores a $41000000 \mathrm{COP}$ que representan un porcentaje superior al $71 \%$ de las ventas del semestre. Así mismo, los meses anteriores revelan los picos más altos de una utilidad. 
Figura 11. Utilidad mensual en el segundo semestre del 2019

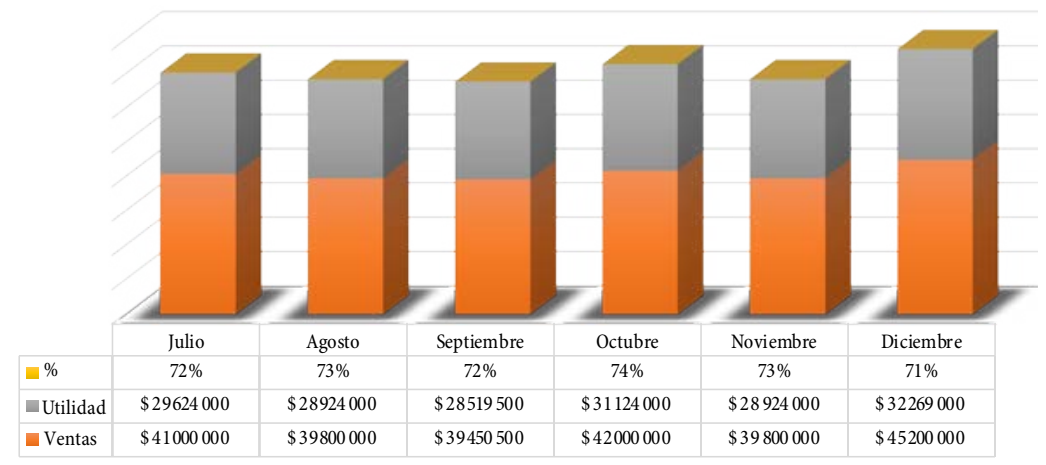

Fuente: elaboración propia.

Con respecto a las compras, la figura 12 demuestra que, durante cuatro meses, estas se mantuvieron constantes en $2000000 \mathrm{cop}$; aunque, en diciembre se dispararon según la tendencia normal de la organización. Asimismo, el $80 \%$ de las compras está relacionado con la materia prima del restaurante.

Figura 12. Comportamiento de las compras

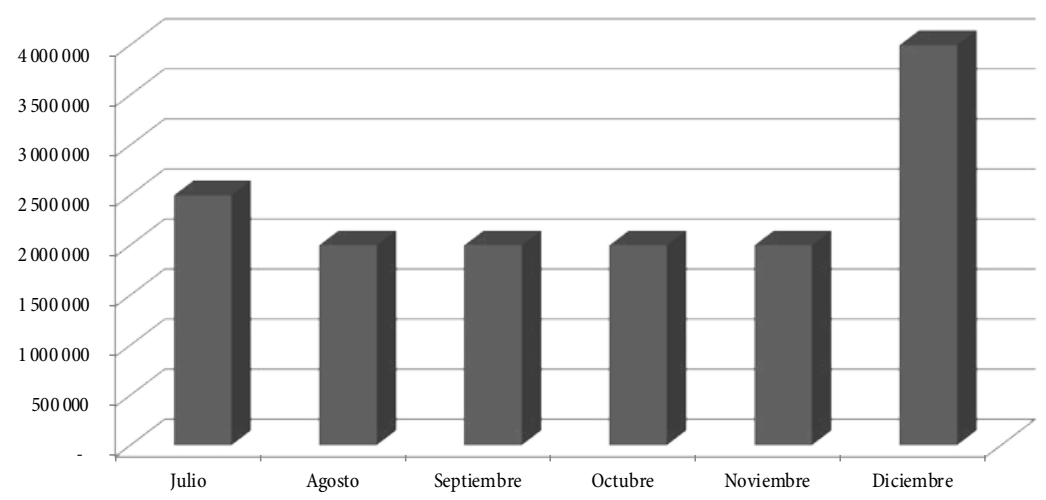

Fuente: elaboración propia. 
Sumado a esto, es importante especificar los gastos administrativos que corresponden al arriendo, los servicios públicos, la telefonía y el impuesto predial, entre otros. Así, la figura 13 ilustra que los gastos mensuales y administrativos son equivalente a los 2200000 cop.

Figura 13. Comportamiento de los gastos administrativos

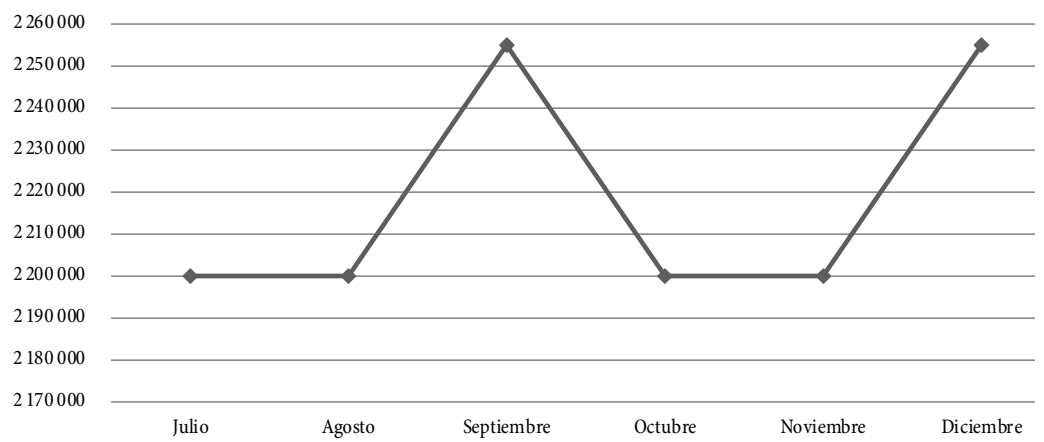

Fuente: elaboración propia.

Finalmente, los gastos por nómina están representados en cuatro trabajadores que laboran cuatro horas diarias en la semana y siete horas el fin de semana. Además, los sueldos dependen del nivel de jerarquía y la actividad que aquellos desempeñan dentro de la empresa; es por esto que el administrador (empleado 1) es quien devenga el mayor sueldo (figura 14). Paralelamente, la figura 15 demuestra que los gastos en los que incurre la compañía son constantes en 6676000 cada mes, puesto que los horarios son fijos y no existen horas extras, ni nocturnas. 
Figura 14. Nómina

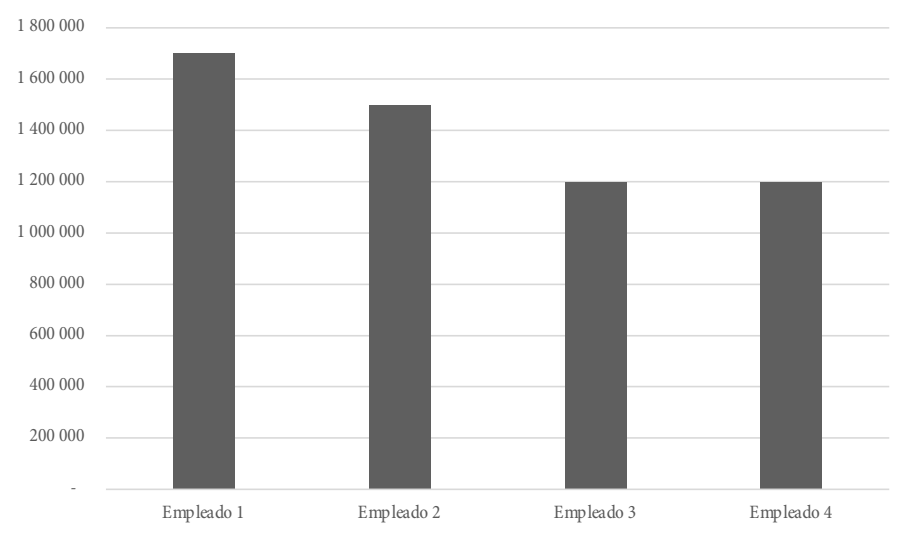

Fuente: elaboración propia.

Figura 15. Gastos mensuales por nómina

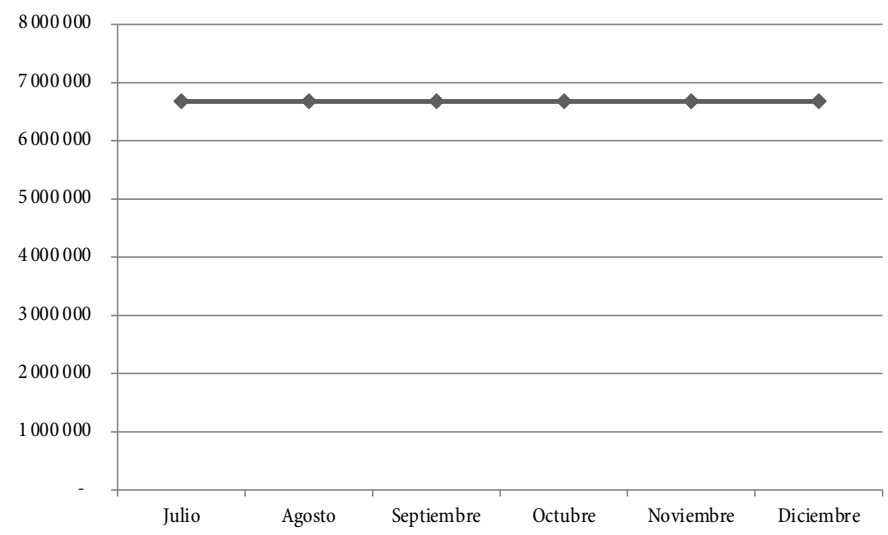

Fuente: elaboración propia.

Llegado a este punto, se logra analizar que la empresa cuenta con una rentabilidad "razonable" debido a la calidad de sus productos. Sin embargo, la falta de políticas y lineamientos en torno al control de los inventarios en Due Amici Pizzería, conlleva a que no se conozca claramente cómo llevar la gestión financiera. Algunos de los problemas identificados son, 
(1) no se establecen tiempos de entrega de los productos hacia los clientes; (2) no existe un estudio de proveedores; (3) no se realiza el cruce de facturas; (4) gran parte de los movimientos no se registran en el software contable que la empresa tiene como herramienta de trabajo; (5) falta de controles de consumo de inventarios a los empleados; y, (6) falta de controles en el servicio de atención al cliente. Todo lo anterior, genera una menor rentabilidad para la organización, por lo que es posible concluir que la inexistencia de controles internos en los inventarios sí incide en la gestión financiera.

\section{Conclusiones}

Gracias a la intervención directa realizada en el restaurante Due Amici Pizzería y de acuerdo con los hallazgos encontrados, se logra concluir que no existen políticas de inventarios para indicar el proceso adecuado de las compras, el almacenamiento y otras fases. Esto se debe a que el $80 \%$ de sus procedimientos se implementan por medio del conocimiento empírico y la experticia del administrador, mientras que el otro $20 \%$ hace una aproximación a la formalización, a pesar de no utilizar un fundamento administrativo o contable.

De igual forma, se determinó que el $65 \%$ de las responsabilidades recaen nuevamente sobre el administrador, quien fue y sigue siendo el encargado de elegir a los proveedores, cuya elección no descansa en según ningún estudio concienzudo debido a la escasez de oferta que existe en el mercado con respecto a los productos requeridos. A causa de esto, el $78 \%$ de sus proveedores vienen acompañándolos desde los inicios del restaurante.

Es importante resaltar que, a pesar de no contar con políticas formales para llevar a cabo el desarrollo de las actividades, la empresa definió una frecuencia de compras semanal a raíz del inventariado que se realiza cada domingo; sin embargo, durante la semana se realizan compras de otros productos que se requieran. 
Dado que las facturas de compra no siempre se cruzan con las órdenes de compra generadas, ha surgido un problema: se obtiene más mercancía de la necesitada y no es posible almacenarla, ya que una gran parte de sus insumos tiene un alto grado de sensibilidad como la harina, los diferentes quesos, las hojas verdes de alto costo, entre otros. En este sentido, se considera necesario ejecutar una reestructuración del control interno de los inventarios y plasmarlo por escrito con el objetivo de tener procesos de planificación y seguimientos que permitan conocer el inventario final y minimizar la pérdida de este.

Además, dentro de las políticas y los procesos, la organización debe preguntarse “qué vamos a hacer?, ¿cómo lo vamos a resolver?", porque no cumple con las políticas de stock de seguridad, ni riesgos por escasez, debido a su poca capacidad de almacenamiento y a los cambios que surgen en las ventas. Lo anterior, permite concluir que Due Amici Pizzería no puede pactar con el proveedor dichos insumos, ni llevar un inventariado selectivo para aquellos insumos de mayor rotación (como lo son los quesos y las diferentes harinas). Adicionalmente, no se manejan niveles de estacionalidad por que los dos productos que requieren del único horno no manejan el mismo nivel de temperatura, revelando así, que no ejecutan el sistema de inventario permanente ni periódico.

Ante la falta de supervisión administrativa en las buenas prácticas laborales que los trabajadores deben ejecutar, los empleados implicados presentan comportamientos ineficientes en el desarrollo de las actividades que repercuten en el inventario: aquellos no usan el software contable, no cruzan facturas, no definen un sistema de inventario a la hora de realizar el conteo físico, entre otras actividades mal ejecutadas. Esto provoca un impacto negativo en la rentabilidad del restaurante, que a su vez es consecuencia de la inexistencia de una gestión financiera dentro de la organización. Dicha situación nos permite reflexionar que tanto el administrador como los socios desconocen la información verídica respecto a la entrada y la salida de los insumos. 
En ese orden de ideas, esta investigación demuestra que existe una incidencia directa en el control interno de los inventarios y la gestión financiera; debido a que es imposible garantizar una rentabilidad eficiente y niveles de competitividad altos, sin procesos de comprobación, intervención o incluso inspección de los inventarios ya que estos activos representan uno de los principales recursos de los que dispone una entidad para la toma de decisiones. En consecuencia, las situaciones internas del restaurante impidieron garantizar los resultados fiables de los inventarios y en los estados financieros de la organización.

Por último, se recomienda que investigaciones futuras retomen el foco del estudio en cuestión, con el propósito de buscar situaciones semejantes que permitan diseñar estrategias o medidas oportunas para el tratamiento de los inventarios, soportados en políticas sólidas y acordes al objeto social de la organización. En ese proceso, los contadores públicos juegan un papel importante y decisivo para el direccionamiento tanto de los emprendedores, como de los pequeños empresarios que ya están en el entorno mercantil.

\section{Referencias}

Aristizabal, C. y Guzmán, Y. (2017). Diseños de los procedimientos de Control Interno para la Gestión de Inventarios de la Comercializadora Je F. (Trabajo de grado). Pontificia Universidad Javeriana. Cali, Colombia.

Ariza, Y. y Villasmil M. (2014). Teoría contable: fundamento de análisis en el ejercicio profesional y la práctica pedagógica, en Desarrollo Gerencial. Revista de la Facultad de Ciencias Económicas, Administrativas y Contables de la Universidad Simón Bolívar, 6(2), 41-71.

Ávila, S. (2010). Guía práctica: logística y distribución física internacional. Cámara de Comercio de Bogotá, Legis S.A.

Biswas, S., Karmaker, C., Islam, A., Hossain, N. y Ahmed, S. (2017). Analysis of Different Inventory Control Techniques: A Case Study in a Retail Shop. Journal of Supply Chain Management Systems, 6(3), 35.

Carvalho, J. (2009). Estado de resultados: procesos contables. Ecoe Ediciones. 
Castellanos, A. (2017). Logística comercial internacional. Editorial Universidad del Norte.

Cepeda, G. (2002). Auditoría y control interno. Editorial Nomos S.A.

Chenhall, R. y Smith, D. (2011). A review of Australian management accounting research: 1980-2009. Accounting \& Finance, 51(1), 173-206.

Congreso de la República de Colombia. (29 de noviembre de 1993). Ley 87. DO: 41.120 .

Cuervo, J., Osorio, J. y Duque, M. (2016). Costeo basado en actividades ABC: Gestión basada en actividades ABM. Ecoe Ediciones.

Díaz, M. P. y Tubón, V. G. (2018). La evaluación del sistema del control interno como soporte estratégico en la gestión de objetivos en las finanzas populares del Ecuador. Apuntes Contables, (21), 117-126.

Gitman, J. (2016). Principios de Administración Financiera. Pearson de México.

Godoy, E. (2014). Normas Internacionales de Información Financiera. Grupo Editorial Nueva Legislación S.A.S.

Hernández, R., Fernández, C. y Baptista, P. (2014). Metodología de la investigación (6 ${ }^{\mathrm{a}}$ ed.). McGraw-Hill.

Kumar, G. A., Anzil, A., Ashik, K., James, A. T., y Ashok, J. K. (2017). Effective Inventory Management system through selective inventory control. Imperial Journal of Interdisciplinary Research, 3(6). 25-39.

Montaño, J., (2015). La Revisoría Fiscal en Colombia: del hacer al deber ser. Editorial Universidad del Valle.

Mora, A. (2016). Inventario cero: Cuándo y cuánto pedir. Alfaomega S. A.

Mora, M. y Espinosa, R. (2018). Gestión financiera con enfoque de procesos en la Universidad Técnica de Manabí. Revista Cubana de Contabilidad y Finanzas, (1), 32-42.

Nahmias, S. (2014). Análisis de la producción y las operaciones. Edamsa Impresiones.

Peña, O. y Oliveira, R. (2016). Factores incidentes sobre la gestión de sistemas de inventario en organizaciones venezolanas. Revista Telos, 18(2), 187-207.

Perdomo, A. (2000). Administración Financiera de Inventarios tradicional y justo a tiempo. Thomson Learning.

Pereira, C. (2019). Actualidad de la gestión empresarial en las pymes. Apuntes Contables, 24, 39-53. https://doi.org/10.18601/16577175.n24.03. 
Ramírez, N. y Ramos, K. (2016). Diseño de un sistema de gestión para el control de inventario en la empresa Electrónica Frank " $R$ ” (Tesis de Pregrado). Universidad de Cartagena, Colombia.

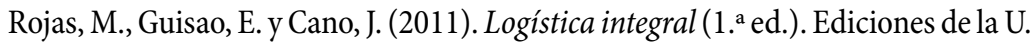
Serna, D. y Rivera, Y. (2018). Dinámica de sistemas en la gestión de inventarios. Ingenierías USBMed, 9(1), 75-85. https://doi.org/10.21500/20275846.3305

Shenoy, D., y Rosas, R. (2018). Inventory Control Systems: Design Factors. En Problems \& Solutions in Inventory Management (pp. 13-32). Springer.

Terrazas, R. (2009). Modelo de Gestión Financiera para una organización. Perspectivas, 12(23), 55-72.

Úbeda, F. (2013). Evaluación del control interno del área de Inventarios en la fábrica de puros ACCSA. Revista Científica de FAREM-Estelí, (7), 21-25. https://doi. org/10.5377/farem.v0i7.2629

Wang, Q., Wu, J., Zhao, N., y Zhu, Q. (2019). Inventory control and supply chain management: A green growth perspective. Resources, Conservation and Recycling, 145, 78-85.

Weber, R., Herold, C., Hollert, H., Kamphues, J., Blepp, M., y Ballschmiter, K. (2018). Reviewing the relevance of dioxin and PCB sources for food from animal origin and the need for their inventory, control and management. Environmental Sciences Europe, 30(1), 42-61.

Zhong, S., Singh, S. K., y Goh, M. (2020). Efficient Supplier Selection: A Way to Better Inventory Control. En Optimization and Inventory Management (pp. 55-279). Springer. 